\title{
Disorder in Josephson-Junction Arrays in a Magnetic Field
}

\author{
Enzo Granato \\ Laboratório Associado de Sensores e Materiais, Instituto de Pesquisas Espaciais, \\ 12201 São José dos Campos, São Paulo, Brazil \\ J. M. Kosterlitz \\ Department of Physics, Brown University, Providence, Rhode Island 02912
}

(Received 4 August 1988)

\begin{abstract}
We consider the effects of disorder in Josephson-junction arrays with an average flux quanta per plaquette $f_{0}$. We show that for $f_{0}=n+\frac{1}{2}$, positional disorder decouples the excitations responsible for the destruction of superconductivity from other excitations so that near the critical field $f_{c}$, the superconducting properties are described by the theory of integer $f_{0}$, leading to the same critical field.

PACS numbers: $74.50 .+\mathrm{r}, 64.60 . \mathrm{Cn}, 74.40 .+\mathrm{k}$
\end{abstract}

Two-dimensional arrays of Josephson junctions have attracted a great deal of attention recently, both experimentally ${ }^{1-3}$ and theoretically. ${ }^{4-13}$ They provide a convenient model system in which the resistive transition can be studied without the large randomness inherent in granular films and, in the presence of a magnetic field perpendicular to the array, allow the study of transitions in frustrated $X Y$ systems. The system of junctions, in the large capacitance limit, is modeled by a frustrated $X Y$ model where the degree of frustration $f$ may be taken to be the number of flux quanta $\phi_{0}=h c / 2 e$ per plaquette of area $S, f=H S / \phi_{0}$. This can be varied experimentally by adjusting the field. The Hamiltonian of the system, in the phase-only approximation

$$
-\beta H=\sum_{\left\langle r r^{\prime}\right\rangle} K_{r r^{\prime}} \cos \left(\theta_{r}-\theta_{r^{\prime}}-A_{r r^{\prime}}\right)
$$

is periodic in $f$ with period one, except for small variations in the coupling constant $K_{r r}$ due to flux penetration of the junction. In Eq. (1), $\theta_{r}$ is the phase of the superconducting grain located at $r, A_{r r^{\prime}}=\left(2 \pi / \phi_{0}\right) \int_{r}^{r^{\prime}} \mathbf{A} \cdot d \mathbf{l}$, and the sum is over nearest neighbors only.

Although a regular array minimizes the effects of disorder, some is inevitably present and should be taken into account. ${ }^{10-12}$ Two kinds of disorder can be present: random plaquette areas and positional disorder. ${ }^{11}$ The former is realized when the superconducting grains have random sizes and the external field is sufficiently low to permit a Meissner effect in an individual grain so that the fluxes are independent random variables. This type of disorder leads to the destruction of superconductivity for dimensions $d<4$ and in $d=2$ to a correlation length $\xi \sim 1 / H$. This does not mean that experimentally there will be no superconductivity because of finite-size effects and vortex pinning by disorder. ${ }^{14}$ In the latter case, positional disorder only, which should be realized with a uniform grain size or $H>H_{c g}$, the lower critical field for an individual grain, quasi-long-range phase coherence and hence superconductivity is found in $d=2$ for a range of temperatures $T^{-}<T<T^{+}$for sufficiently low fields.
Recent experiments on proximity-coupled arrays with deliberate positional disorder ${ }^{3}$ are consistent with the theoretical predictions ${ }^{11}$ for integer values of $f_{0}$, the average number of flux quanta per plaquette. The maximum field for which superconductivity is possible is predicted to be $f_{c} \Delta \leq 1 /(32 \pi)^{1 / 2}$ where the probability distribution of the grain positions is $P(\mathbf{u}) \propto \exp \left(-\mathbf{u}_{r}^{2} / 2 \Delta^{2}\right)$, where $\mathbf{u}_{r}$ is the displacement of a grain from its average position $\mathbf{r}$. The inequality is due to small corrections due to finite fugacity effects. Agreement between theory and experiment is obtained when one extrapolates the experimental data for $f_{0}=n+p / q$ to large values of $q$ since resistance measurements for $f_{0}=n$ probe length scales of the order of a lattice spacing $a_{0}$, and for $f_{0}(q)=n+p / q$ length scales of order $q a_{0}$. The theoretical prediction is for infinite length scales so such an extrapolation is necessary. However, this makes sense only if the theoretical critical value $f_{c}(q)$ is independent of $q$, which is counterintuitive because of effects which may be important for $q>1$, such as domain walls, fractional charges, etc. ${ }^{8}$ The only reason why such a hypothesis could be true is that a finite amount of positional disorder ensures that such objects are decoupled from integer valued charges (vortices) which unbind first. These correspond to isolated defects in the superlattice ground state. $^{4,7,8}$ If such a scenario is correct, then the breakdown of superconductivity is governed by the same mechanism as for $f_{0}=n$.

In this Letter, we argue on the basis of a specific model, that positional disorder does, in fact, decouple the integer charges from the other excitations at least for $f_{0}=n+\frac{1}{2}$. Although we have not been able to demonstrate it, we believe that this is also true for other rational values of $f_{0}$. The physics behind this surprising effect relies on two facts. First, the pure system has a $Z_{2}$ symmetry as well as the usual U(1) symmetry and Ising order can coexist with $X Y$ disorder but not vice versa. ${ }^{7,8}$ Thus, as temperature is raised, an $X Y$ transition occurs first followed by an Ising one. The alternative scenario is 
a single transition in which Ising disorder causes $X Y$ disorder. The nature of this is not known but the mechanism in the isotropic-junction array is that at corners of domains of Ising order there are fractional charges which unbind when the domain walls melt which then screen the integer charges. The result is a simultaneous loss of both chiral (Ising) order and superconductivity. ${ }^{7,8}$

The second piece of physics is that positional disorder of the grains affects the $X Y$ order in a much more dramatic way than the Ising order. Thus, increasing the amount of disorder will reduce the $X Y$ transition temperature to below that of the Ising and will eventually allow the double transition scenario. At this stage, the superconductivity is controlled only by the integer charges, thereby mapping the system on to the theory of logarithmically interacting charges in a random dipolar background $^{11,15}$ which leads to a critical field $f_{c} \Delta$ $\leq 1 /(32 \pi)^{1 / 2}$. When the $X Y$ degrees of freedom disorder, the Ising order persists but does not qualitatively affect the superconducting transition. The possible phase diagrams are shown in Fig. 1 and in both cases the maximum field is the same. ${ }^{16}$

The calculations leading to these conclusions for $f_{0}=n+\frac{1}{2}$ are summarized in the remainder of this Letter. One first observes that disorder can be incorporated in the phase-only approximation of Eq. (1) via $A_{r r^{\prime}}=A_{r r^{\prime}}^{0}+2 \pi f_{0} t_{r r^{\prime}}$. Summing over the links $r r^{\prime}$ surrounding the site $R$ of the dual lattices yields $\sum A_{r r^{\prime}}^{0}=2 \pi f_{0}$ and $\sum t_{r r^{\prime}}=\delta f_{R} / f_{0}$. In the case of random flux per plaquette, $\delta f_{R}$ is an independent random variable, and in the case of positional disorder only, the in-

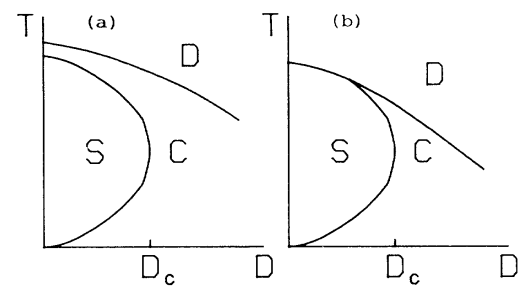

FIG. 1. Possible phase diagrams for a Josephson-junction array with $f_{0}=n+\frac{1}{2}$ as a function of disorder $D=\Delta f_{0}$. S, C, and $\mathrm{D}$ denote the chirally ordered superconducting, chirally ordered normal, and disordered phases, respectively. In (a) two separate transitions occur and in (b) a single transition occurs when $\Delta=0$. In both cases phase coherence is possible only for $D \leq(32 \pi)^{1 / 2}$.

dependent random variables are $\mathbf{u}_{r}$. In the latter case

$$
t_{r r^{\prime}}=\hat{\mathbf{z}} \cdot\left(\mathbf{r}-\mathbf{r}^{\prime}\right) \times\left(\mathbf{u}_{r}+\mathbf{u}_{r^{\prime}}\right) / 2 a_{0}^{2} .
$$

The strategy is to convert Eq. (1) into a Coulomb-gas representation. It is known that for the $f_{0}=n+\frac{1}{2}$ model without disorder, the ground state is an antiferromagnetic arrangement of $\pm \frac{1}{2}$ charges on the dual lattice and that the lowest energy excitations are walls separating the two degenerate ground states, $\pm \frac{1}{4}$ charges at domain wall corners and isolated regions of unit excess charges interacting logarithmically. The Coulomb-gas Hamiltonian can be written in terms of Ising variables $S_{R}= \pm 1$ and charges $Q_{r}=\Sigma_{\mathrm{e}} S(\mathrm{r}+\mathrm{e}) / 8$ with e being the vectors from the center of a plaquette to the four corners, ${ }^{13}$

$$
\beta H=L_{1} \sum_{\left\langle R R^{\prime}\right\rangle} S_{R} S_{R^{\prime}}+2 \pi^{2} K \sum_{r r^{\prime}} Q_{r} G\left(r-r^{\prime}\right) Q_{r^{\prime}}+L_{2} \sum_{R} S_{R} \delta f_{R}+4 \pi^{2} K \sum_{r R} Q_{r} G(r-R) \delta f_{R},
$$

where $G(r)$ is the Coulomb-gas interaction. The Ising variables, which are just twice the charge at the site, are coupled antiferromagnetically by a short-range interaction represented by a nearest-neighbor coupling $L_{1}$ and to the random $\delta f_{R}$ by another short-range interaction represented by a local coupling $L_{2}$. Note that in an array, there is no particular reason why $L_{1}$ and $L_{2}$ should be related in any simple way to the coefficient $K$ of the long-range part. This is because they depend crucially on the short-range parts of the interactions between the original charges of the model in contrast to the corner and excess integer charges.

In the case where $\delta f_{R}$, the deviation of the flux, is an independent random variable it is clear that $\delta f_{R}$ acts like a random magnetic field on the Ising variables and like a random charge on the corner and excess charges. This will then cause loss of both chiral order and superconductivity for any disorder. ${ }^{11,17}$

In the absence of randomness in the flux, all that is left is positional disorder and the last two terms describing the disorder in the Hamiltonian of Eq. (3) become

$$
\beta H_{\mathrm{dis}}=L_{2} f_{0} \sum_{\left\langle r r^{\prime}\right\rangle} t_{r r^{\prime}}\left(S_{R}-S_{R^{\prime}}\right)+4 \pi^{2} K f_{0} \sum_{\left\langle r r^{\prime}\right\rangle} \sum_{r^{\prime \prime}} t_{r r^{\prime}} Q_{r^{\prime \prime}}\left[G\left(R-r^{\prime \prime}\right)-R\left(R^{\prime}-r^{\prime \prime}\right)\right],
$$

where the bond $\left\langle R R^{\prime}\right\rangle$ on the dual lattice cuts the bond $\left\langle r r^{\prime}\right\rangle$ on the original with $R$ to the left and $R^{\prime}$ to the right of $\left\langle r r^{\prime}\right\rangle$. Using Eq. (2), it is easy to show that the first term of Eq. (4) corresponds to a local coupling of the displacement $\mathbf{u}_{r}$ to a domain wall. This is equivalent to random bond disorder. ${ }^{18}$ The last term corresponds to the coupling of the charges $Q$ to a random dipole background. One expects that the Ising variables $S_{R}$ are much less affected by the random bond disorder than are the integer charges which correspond to small domains with unit of excess charge. These are not attached to domain walls but interact logarithmically with each other and with the random dipole background. At sufficiently large disorder, the transition bifurcates as discussed earlier leading to our claimed result. 
This last part of the argument is somewhat weak since we have been unable to justify it by actual calculation on this model. However, a related model derived from a Ginzburg-Landau expansion of the original one is in the same universality class ${ }^{6,9}$ and has the same phase diagram in the absence of disorder which supports these conclusions. In the present case, a similar expansion about the most strongly fluctuating modes $\Phi_{i}=\left|\Phi_{i}\right| e^{i \phi_{i}}$ leads to an effective Hamiltonian of the form

$$
\beta H=-K \sum_{\left\langle r r^{\prime}\right\rangle} \sum_{i=1}^{2} \cos \left(\phi_{i r}-\phi_{i r^{\prime}}-2 \pi f_{0} t_{r r^{\prime}}\right)+g \sum_{\left\langle r r^{\prime}\right\rangle} \cos \left(\phi_{1 r}-\phi_{2 r}-\phi_{1 r^{\prime}}+\phi_{2 r^{\prime}}\right)-h \sum_{r} \cos 2\left(\phi_{1 r}-\phi_{2 r}\right) .
$$

Note that all these terms are allowed by symmetry. ${ }^{7}$ The last term reflects the existence of domain walls. One can construct a Coulomb-gas representation and one finds, as expected, that the coupling between the two phases $\phi_{1}$ and $\phi_{2}$ is very relevant. The $h \rightarrow \infty$ strong coupling limit implies that $\phi_{2 r}=\phi_{1 r}+\pi \tau_{r}$, with $\tau_{r}=0,1$. The action can now be written as $\left(\sigma_{r}=2 \tau_{r}-1\right)$

$$
\begin{aligned}
\beta H= & -K \sum_{\left\langle r^{\prime}\right\rangle} \cos \left(\phi_{r}-\phi_{r^{\prime}}-2 \pi f_{0} t_{r r^{\prime}}\right)\left(1+\sigma_{r} \sigma_{r^{\prime}}\right) \\
& +g \sum_{\left\langle r r^{\prime}\right\rangle} \sigma_{r} \sigma_{r^{\prime}} .
\end{aligned}
$$

From Eq. (6) it is clear that the system will be completely disordered when Ising order is destroyed. The system breaks up into finite domains of Ising order and, since there is no coupling of $\phi$ across domain walls, phase coherence is also lost by a transition of unknown type. The loss of Ising order is controlled by $K-g$ and the $X Y$ transition by $K$ so that even when there is no disorder $\left(\Delta f_{0}=0\right)$, a single transition will occur for $g>g_{c}$ and a double one for $g<g_{c}$ with the $X Y$ occurring first. ${ }^{13}$ When $g \ll 0$, it is clear that the Ising order will persist with $X Y$ disorder. Now imagine that the system has Ising order so that $\phi$ is controlled by an effective action

$$
\beta H_{\mathrm{eff}}=-\tilde{K} \sum \cos \left(\phi_{r}-\phi_{r^{\prime}}-2 \pi f_{0} t_{r r^{\prime}}\right),
$$

with $\tilde{K} \simeq K\left(1+\langle\sigma\rangle^{2}\right)$. Using standard methods, ${ }^{11,15}$ one can construct recursion relations for $\tilde{K}$ and the vortex fugacity $y$. These show that there is a range of $\tilde{K}$ for which $y$ is irrelevant which implies phase coherence;

$$
d y / d l=\left(2-\pi \tilde{K} 4 \pi^{3} \Delta^{2} f_{0}^{2} \tilde{K}^{2}\right) y .
$$

From Eq. (7) one sees that $\pi \tilde{K}_{X Y}^{c}\left(\Delta f_{0}\right)$ lies in the range $2<\pi \tilde{K}_{X Y}^{c}\left(\Delta f_{0}\right)<4$, the lower value for $\Delta f_{0}=0$ and the larger for the upper bound on the critical field $\Delta f_{c}=1 /(32 \pi)^{1 / 2}$. A reentrant transition is also predicted. ${ }^{11,15}$ The actual critical field will be somewhat less than this. ${ }^{15}$ Since the final disordering temperature depends on both $K$ and $g$, it is easy to imagine a situation in which $K_{I}^{c}>K_{X Y}^{c}(0)$ but $K_{I}^{c}<K_{X Y}^{c}\left(\Delta f_{0}\right)$ with $f_{0}<f_{c}$. In such a case, the transition will be single for small $f_{0}$ but splits for larger $f_{0}$. The final transition will be that of a weak random bond Ising ferromagnet ${ }^{18}$ with specific heat $C \sim \ln \ln t$. Although not conclusive, Monte Carlo data ${ }^{10}$ are consistent with these ideas since the observed specific heat peak is at a $f_{0}$-independent temperature and, as $f_{0}$ increases, just fades away. Better statistics are needed to see any splitting.

In general, to describe the $X Y$ transition in the double region, we should imagine first rescaling up to the Ising correlation length $\xi_{I}$ so that the upper bound will be $\Delta\left(\xi_{I}\right) f_{c}=1 /(32 \pi)^{1 / 2}$. We have checked the above scenario from the Coulomb-gas representation of Eq. (5) and the small fugacity recursion relations which lead to the same picture. These also show that $\Delta$ does not rescale since it always appears in the combination $K^{\prime}=4 \pi^{2} \Delta^{2} f_{0}^{2} K^{2}$ which is preserved under rescaling. Hence, $f_{c}$ for the frustrated array is, to within small corrections, the same as in the unfrustrated case.

One may object to the conclusions about an array based on the $X Y$-Ising system of Eq. (6) which does not contain domain-wall corner charges. This will make no difference in the double transition region since at the $X Y$ transition there are no domain walls. Even in the original model it is physically very plausible that the disorder will cause a dramatic reduction in $T_{c}$ for the $X Y$ part but not much for the Ising part, thus implying a splitting of the single transition into two. The single transition may be different because of the different mechanisms by which loss of Ising order causes simultaneous loss of $X Y$ order. The two models have Ginzburg-Landau expansions which differ only by irrelevant operators. Monte Carlo simulations show that they have the same topology of phase diagram and the only place where we used the modified model was to argue that the single transition splits.

It is believed that the $f_{0}=n+\frac{1}{2}$ junction array in the absence of disorder lies very close to the bifurcation point ${ }^{13}$ so that even small disorder well below its critical value will induce the bifurcation. Therefore the estimate $f_{c} \Delta \simeq 1 /(32 \pi)^{1 / 2}$ should be rather good for both integer an half integer $f_{0}$. Similar considerations very likely hold for $f_{0}=n+p / q$ but this is just speculation. The considerations of this Letter also have implications for the dynamics of arrays at $f_{0}=\frac{1}{2}$ in that the crossover from inductive to resistive behavior may well be described by the dynamic theory of the unfrustrated case, which seems to be the case. ${ }^{19}$

We thank C. J. Lobb and P. Martinoli for useful discussions. E.G. gratefully acknowledges the support from Fundacão de Amparo e Pesquisa do Estado de São Paulo (FAPESP) and Coordenacão de Aperfeicoamento de Pessoal do Ensino Superior (CAPES) (Brazilian agencies) and J.M.K. from the NSF through Grant No. DMR-8611455. One of us (J.M.K.) acknowledges FAPESP for supporting a visit to the Instituto de Pesquisas Espaciais where part of this work was done. $\mathrm{He}$ 
thanks the members of that Institute for their hospitality.

${ }^{1}$ R. F. Voss and R. A. Webb, Phys. Rev. B 25, 3446 (1982); R. A. Webb, R. F. Voss, G. Grinstein, and P. M. Horn, Phys. Rev. Lett. 51, 690 (1983); B. J. van Wees, H. S. J. van der Zant, and J. E. Mooij, Phys. Rev. B 35, 7291 (1987).

${ }^{2}$ M. Tinkham, D. W. Abraham, and C. J. Lobb, Phys. Rev. B 28, 6578 (1983); D. Kimhi, F. Leyraz, and D. Ariosa, Phys. Rev. B 29, 1487 (1984); R. K. Brown and J. C. Garland, Phys. Rev. B 33, 7827 (1986); Ch. Leemann, Ph. Lerch, G.-A. Racine, and P. Martinoli, Phys. Rev. Lett. 56, 1291 (1986); J. P. Carini, Phys. Rev. B 38, 63 (1988).

${ }^{3}$ M. G. Forrester, H. J. Lee, M. Tinkham, and C. J. Lobb, Jpn. J. Appl. Phys. 26, 1423 (1987), and Phys. Rev. B 37, 5966 (1988). The main features such as the decay with increasing disorder of the amplitude of resistance oscillations are governed by short-length scales which is well described by mean-field theory; S. P. Benz, M. G. Forrester, M. Tinkham, and C. J. Lobb, Phys. Rev. B 38, 2869 (1988).

${ }^{4}$ S. Teitel and C. Jayaprakash, Phys. Rev. Lett. 51, 199 (1983), and Phys. Rev. B 27, 598 (1983).

${ }^{5}$ B. Berge, H. T. Diep, A. Ghazali, and P. Lallemand, Phys. Rev. B 34, 3177 (1986); W. Y. Shih and D. Stroud, Phys. Rev. B 32, 158 (1985); J. E. van Himbergen, Phys. Rev. B 33, 7857 (1986).

${ }^{6}$ M. Y. Choi and S. Doniach, Phys. Rev. B 31, 4516 (1985); M. Y. Choi and D. Stroud, Phys. Rev. B 32, 5773 (1985).
${ }^{7}$ M. Yosefin and E. Domany, Phys. Rev. B 32, 1778 (1985); T. C. Halsey, J. Phys. C 18, 2437 (1985).

${ }^{8}$ T. C. Halsey, Phys. Rev. B 31, 5728 (1985); S. E. Korshunov and G. V. Uimin, J. Stat. Phys. 43, 1 (1986); E. Granato, J. Phys. C 20, L215 (1987).

${ }^{9}$ E. Granato and J. M. Kosterlitz, J. Phys. C 19, L59 (1986), and Phys. Rev. B 33, 4767 (1986).

${ }^{10}$ M. Y. Choi, J. S. Chung, and D. Stroud, Phys. Rev. B 35, 1669 (1987); A. Chakrabarti and C. Dasgupta, Phys. Rev. B 37, 7557 (1988).

${ }^{\prime \prime}$ E. Granato and J. M. Kosterlitz, Phys. Rev. B 33, 6533 (1986).

12 J. M. Kosterlitz and E. Granato, Phys. Rev. B 34, 2026 (1986), and Physica (to be published).

${ }^{13}$ J. M. Thijssen and H. J. F. Knops, Phys. Rev. B 37, 7738 (1988).

${ }^{14}$ A. I. Larkin and Yu. N. Onchinnikov, J. Low Temp. Phys. 34, 409 (1979); D. S. Fisher, Phys. Rev. B 22, 1190 (1980); P. Martinoli, M. Nsabimona, G. A. Racine, H. Beck, and J. R. Clem, Helv. Phys. Acta 56, 765 (1983); J. C. Garland and H. J. Lee, Phys. Rev. B 36, 3683 (1987).

${ }^{15}$ M. Rubinstein, B. Shraimain, and D. R. Nelson, Phys. Rev. B 27, 1800 (1983).

${ }^{16}$ At very low temperatures one expects a nonequilibrium glassy phase due to vortex pinning which masks the reentrant transition in Monte Carlo simulations and experiments.

${ }^{17}$ J. Z. Imbrie, Phys. Rev. Lett. 52, 174 (1984).

${ }^{18}$ V. S. Dotsenko and V. S. Dotsenko, Adv. Phys. 32, 129 (1983); R. Shankar, Phys. Rev. Lett. 58, 2466 (1987).

${ }^{19} \mathrm{P}$. Martinoli, private communication. 